\title{
28 Research Square \\ Aptamer Based Point of Care Diagnostic for the Detection of Food Allergens
}

\section{Sarah Stidham}

DOTS Technology Corp

Valerie Villareal

DOTS Technology Corp

Vasant Chellappa

DOTS Technology Corp

Lucas Yoder

DOTS Technology Corp

Olivia Alley

DOTS Technology Corp

\section{Wayne Shreffler}

Massachusetts General Hospital

Jonathan Spergel

Children's Hospital of Philadelphia

David Fleischer

Children's Hospital Colorado

Hugh Sampson

Mount Sinai Hospital

Adi Gilboa-Geffen ( $\sim$ agilboa@dotscorp.com)

DOTS Technology Corp

\section{Research Article}

Keywords: Care Diagnostic, Food Allergens, peanut protein

Posted Date: November 18th, 2021

DOI: https://doi.org/10.21203/rs.3.rs-1060991/v1

License: (9) This work is licensed under a Creative Commons Attribution 4.0 International License.

Read Full License 


\section{Abstract}

Aptamers, due to their small size, strong target affinity, and ease of chemical modification, are ideally suited for molecular detection technologies. Here, we describe successful use of aptamer technology in a consumer device for the detection of peanut antigen in food. The novel aptamer-based protein detection method is robust across a wide variety of food matrices and sensitive to peanut protein at concentrations as low as $12.5 \mathrm{ppm}$ ( $37.5 \mu \mathrm{g}$ peanut protein in the sample). Integration of the assay into a sensitive, stable, and consumer friendly portable device will empower users to easily and quickly assess the presence of peanut allergens in foods before eating. With most food reactions occurring outside the home, the type of technology described here has significant potential to improve lives for children and families.

\section{Introduction}

Aptamers are oligonucleotides capable of high-affinity binding to target molecules ${ }^{1}$. Since development of the in vitro systematic evolution of ligands by exponential enrichment (SELEX) selection process in 1990, aptamers have been designed to selectively bind diverse targets, including RNA, DNA, and other small molecules and compounds ${ }^{2-4}$. These use cases have supported their development as valuable tools for fundamental research, therapeutic applications, and as sensors in molecular diagnostic devices ${ }^{3,5}$. Due to their high affinity, small size, and ease of chemical modification, aptamers have been suggested as a superior reagent for molecular target recognition. They have also gained traction in several clinical applications, with the first aptamer-based therapeutic gaining FDA approval in $2004^{6}$.

The conventional method of detecting antigen is through antibody recognition. Approaches, such as enzyme linked immunosorbent assays (ELISA), immunoblots, visualization with microscopy, etc., indirectly detect targets via primary and secondary antibody recognition and are visualized using fluorophores or active enzymes. The major disadvantages of using antibodies include batch-to-batch variations, hybridoma clone stability, and the time required to produce large scale quantities ${ }^{7,8}$. In contrast, aptamers are chemically synthesized with accuracy and can be stored for long periods of time post-synthesis. They can also be precisely and reproducibly modified with labels such as fluorophores or nucleic acid analogues. Through the intensive SELEX process, aptamers can exhibit high affinity and specificity comparable to monoclonal antibodies ${ }^{5}$ and can recognize diverse antigen types, including DNA, RNA, proteins, and cells $s^{9,10}$.

Antigen detection approaches such as ELISA that rely on antibodies have additional disadvantages, including multistep processing and the requirement for large or expensive equipment and trained personnel to perform experimental procedures and interpret results. This confines these approaches to laboratory settings and increases time and cost. Here we address the need for simple, rapid antigen detection by demonstrating a fluorophore-labeled aptamer-based assay for the detection of allergenic peanut proteins and have incorporated it into an easy to use, point-of-care device suitable for consumer use. Detection of peanut protein was chosen due to the large population of people allergic to peanut 
(Arachis hypogaea) ${ }^{11}$; up to $2.2 \%$ of the US population has an allergy to peanut according to recent epidemiological studies $^{12}$. Given that most food reactions occur upon consumption of food outside of the home ${ }^{11-13}$, we address consumers' need to test for the presence of peanut protein in foods by creating a portable and robust assay capable of sensitive and specific antigen detection in complex and variable matrices (i.e., foods) ${ }^{14}$.

\section{Results}

\section{Aptamer Selection}

Five aptamers were initially chosen (P1-16, P1-10, PT-31, P2-8, and P2-18), based on a peanut-targeted SELEX pool, and underwent sequence modifications to improve tertiary structure formation using the predicted change in Gibbs free energy ${ }^{1,15}$. To develop an aptamer-based assay with an optical readout, the aptamers were conjugated with a Texas Red fluorophore on the 5' end.

To determine the affinity of each optimized aptamer for Ara h 1, a major peanut allergen and the most abundant allergen in peanut (12-16\% of the total protein content) ${ }^{16,17}$, increasing amounts of purified unlabeled Ara h 1 were incubated with each aptamer and analyzed using fluorescence polarization (FP) to screen for binding affinity (Figure 1A). The TR-labeled P1-16 aptamer yielded the highest affinity for Ara $\mathrm{h} 1\left(\mathrm{~K}_{\mathrm{d}} \sim 54 \pm 5.5 \mathrm{nM}\right)$ followed by P1-10 and P2-18 (SI Table 1).

To determine whether the aptamers could detect the presence of Ara $\mathrm{h} 1$ in processed peanut, we incubated the aptamers with commercially available peanut flour or peanut butter and repeated the affinity measurements. As observed with purified Ara $\mathrm{h}$ 1, the TR-labeled P1-16 aptamer yielded a higher affinity for Ara $\mathrm{h} 1$ in peanut butter and peanut flour $\left(\mathrm{K}_{\mathrm{d}} \sim 141 \pm 21.9 \mathrm{ppm}\right.$, and $144 \pm 31.4 \mathrm{ppm}$, respectively) when compared to the other four aptamers (Figure 1B-C). Protein, the allergy triggering component, makes up $25 \%$ of the peanut commodity and measured by ELISA, the amount of accessible Ara $\mathrm{H} 1$ in the peanut flour was $1.8 \%$ by mass (SI Figure 1 ).

\section{Assay Design}

FP is sensitive to viscosity, temperature, and motion effects, and can be affected by auto-fluorescence of the test matrix ${ }^{18,19}$. To overcome these limitations, we designed a robust assay utilizing short complementary sequences ("anchors") that are attached to a solid support (Figure 2). In this assay, the fluorescently labeled aptamer is incubated with the food sample to be tested and, subsequently, applied to the solid support with immobilized anchor sequences. If the aptamer is bound to peanut antigen, it cannot bind to the anchor, and is removed during a subsequent washing step. High fluorescence detected on the support surface therefore signals the absence of peanut antigen (labeled aptamer binds the anchor), and low fluorescence occurs when peanut antigen is present (labeled aptamer is not bound to the anchor). 
To select the best anchor sequence for the application, 40 short DNA sequences (anchors), complementary to various regions of the P1-16 aptamer, were covalently attached to an optically clear glass surface. They differed by oligonucleotide sequence, length, or composition/length of the linker adjacent to the surface (carbon atoms or poly-A tail). To reduce the probability of interference due to matrix auto-fluorescence, the aptamers were conjugated to a Cyanine 5 (Cy5) fluorophore rather than Texas Red. After incubation of Cy5-labeled aptamers with homogenized peanut flour, the peanut flouraptamer mix was added to wells containing the 40 complementary anchors immobilized to glass. After incubation and washing, we detected a decrease in Cy 5 fluorescence associated with an anchor complementary to a loop region of the P1-16 aptamer. Dilution experiments showed the signal was dependent on the concentration of peanut flour with sensitivity as low as $50 \mathrm{ppm}$ peanut flour (equivalent to $12.5 \mathrm{ppm}$ peanut protein). The poly-A linker was determined to increase the sensitivity as well as the fluorescent baseline (SI Figure 2). These results were replicated when the anchor was extended with an additional six carbons, suggesting the positioning of the anchor relative to the glass surface influences aptamer binding.

Functionality of the assay using the selected anchor was confirmed by determining specificity of the P116 aptamer to various Ara h proteins. Consistent with the FP data, fluorescent intensity of P1-16 decreased with increasing concentrations of Ara $\mathrm{h} 1$ and Ara $\mathrm{h} 3$ but not other peanut proteins (Ara $\mathrm{h} 2$, Ara h 6, and Ara h 8) (Figure 3). The finding that P1-16 binds to both Ara $\mathrm{h} 1$ and Ara $\mathrm{h} 3$ is not surprising given that these proteins are members of the cupin superfamily and are structurally similar, with a root mean square deviation (r.m.s.d) of only $2.4 \AA$ when their crystal structures are aligned ${ }^{20-23}$.

To transform the technology into a suitable tool for use by allergic individuals to help manage food consumption choices, we integrated the assay into a small single-use reaction capsule that is run on a durable instrument (SI Figure 3a). The device was designed to receive small food samples $(0.1 \mathrm{~g})$ in a capsule containing the P1-16 aptamer and homogenization buffer (see Methods). The capsule homogenizes samples with the buffer to extract peanut proteins with a small blender, and then passes the homogenate through a polyethylene terephthalate (PET) mesh filter to remove large particulates. The filtered homogenate then flows, via a fluidic sequence, through a reaction chamber (SI Figure 3b), where the anchor sequences are bound. After rapid incubation ( $1-5$ minutes, variable by sample), the aptamer-homogenate mixture is washed away, and the reaction chamber is imaged by a camera in the instrument. An image analysis algorithm detects and interprets fluorescence from the remaining bound aptamer and produces the result that indicates whether peanut was or was not detected in the sample.

To improve robustness of the assay, we designed and tested approaches to normalize the fluorescent signal to an internal control. First, we searched for a control anchor sequence complimentary to a second region of the P1-16 aptamer whose binding would not be sensitive to peanut concentration. To do this, we revisited the 40 sequences in our initial screen (SI Figure 2). Sequences that were complementary or proximal to the covalent anchor or were sensitive to peanut were eliminated. Additional anchor sequences were designed to shift the control anchor sequence farther from the anchor-P1-16 binding site. Most anchors exhibited some reduction in fluorescence intensity in the presence of peanut; however, one 
anchor sequence exhibited only a modest non-significant decrease in intensity when peanut flour up to $200 \mathrm{ppm}$ (equivalent to $50 \mathrm{ppm}$ peanut protein) was introduced. These results suggested that this sequence may function as a matrix condition control (Figure 4A). We immobilized spots of the insensitive "control" anchors and peanut-sensitive "test" anchors in a checkerboard pattern (Figure 4B) on the solid surface of the reaction capsule. This arrangement was chosen to compensate for debris or uneven flow and/or illumination of the reaction chamber (SI Figure 4). The detection algorithm was adjusted to image only after a minimum fluorescence was reached in the control spots. The fluorescent intensities of the test and control spots were then averaged individually and the difference in intensities between the control and test was then normalized ( 1 - intensity of test/intensity of control) to yield a single value comparable across a variety of food matrices.

As a final step to achieve a brighter signal and improved image analysis, we switched the aptamer fluorophore from Cy 5 to Alexa Fluor 647 (AF647). A significant concern with aptamers is stability of the three-dimensional conformation over time. Accelerated aging studies on the AF647 modified P1-16 aptamer at $37^{\circ} \mathrm{C}$ (SI Figure 5) showed that the aptamer retained function over a period of at least three years (68 weeks real time).

\section{Assay Performance}

As this assay was designed to detect peanut protein, we challenged the assay by testing the P1-16 aptamer against multiple types of tree nuts to gauge reactivity towards foods containing proteins of the cupin superfamily ${ }^{25}$. Commercially available almond (Prunis dulcis), cashew (Anacardium occidentale), hazelnut (Corylus avellana), pecan (Carya illinoinensis), pistachio (Pistacia vera), sunflower (Helianthus annuus), and walnut (Juglans regia) flours were tested at $0 \mathrm{ppm}$ and $50 \mathrm{ppm}$ and compared to peanut flour. The amount of protein in the nut flours differs by variety, so concentration of the commodity was held constant. The normalized difference between the test and control spots decreased in the presence of $50 \mathrm{ppm}$ of the tree nut flour tested (Figure 5A), which could indicate significant cross-reactivity. To test whether the specificity of AF647-P1-16 for Ara $\mathrm{h}$ 1/Ara $\mathrm{h} 3$ is greater compared to other cupin family proteins, we added $0.1 \%$ non-fat dry milk to the assay to serve as a nonspecific protein food matrix. The decrease of the test panel compared to the control panel was significantly greater in the presence of peanut when compared to the tree nuts tested in the presence of $0.1 \%$ milk (Figure 5B). We also performed a competition experiment by spiking peanut flour with the same concentration of tree nut flours to gauge whether peanut protein could compete with tree nut protein for P1-16 binding (Figure 5C). The clear distinction in normalized difference between samples that contain peanut and those that do not suggested that $\mathrm{P} 1-16$ is responsive to peanut protein in the presence of tree nut.

A robust assay retains sensitivity regardless of the matrix analyzed, therefore, we performed a guard band study on individual ingredients to investigate the effects of potentially high-risk food components and additives (e.g., fats, acid). Tests were conducted at the highest level of these potentially deleterious matrix components typically seen in foods as reported by the USDA in the presence and absence of 12.5 ppm peanut protein (SI Table 2). As shown in Figure 6A, assay performance was unaffected by many of the components tested, including common sweeteners, insoluble fiber, fats, food coloring, salts, and 
tannins. Variable effects were detected in the presence of acids and alginate (a common thickening agent). High levels of acidity (e.g., pure white vinegar) inhibited the binding of P1-16 to the control panel, to such an extent that a minimum viable signal was never reached. Sodium alginate is tolerated in the assay as high as $0.01 \%$, though alginate can be found in a few foods at higher concentrations, including ice cream, which interestingly does perform well in our assay (see Figure 6B).

To confirm the accuracy of our integrated device, we tested 50 foods that represent 14 food categories suggested by AOAC International for quantitation of peanut by ELISA-based methods as well as foods identified by peanut allergic individuals as high risk for peanut contamination. For each food, at least four replicates were run without peanut (0 ppm) and at least four replicates were spiked with peanut protein (12.5 ppm, equivalent to $37.5 \mu \mathrm{g}$ of peanut protein in a $100 \mathrm{mg}$ food sample). Additionally, we tested 20 commercially available foods that were known to contain peanut. For each food, the normalized difference (1 - test/control) was plotted (Figure 6B and SI Table 3, 4). After setting a threshold value for samples that contain peanut and those that do not, the ability of the assay to detect peanut was confirmed, with an accuracy of $99 \%$ (Table 1 ).

Table 1

Results from 495 runs across 50 foods.

\begin{tabular}{|lll|}
\hline & Number of Runs & $\%$ of Runs \\
\hline True Positive & 232 & $99 \%$ \\
\hline False Negative & 2 & $1 \%$ \\
\hline True Negative & 239 & $98 \%$ \\
\hline False Positive & 5 & $2 \%$ \\
\hline Sensitivity & $99 \%$ & \\
\hline Specificity & $98 \%$ & \\
\hline Accuracy & $99 \%$ & \\
\hline PPV (Positive Predictive Value) & $98 \%$ & \\
\hline NPV (Negative Predictive Value) & $99 \%$ & \\
\hline Likelihood ratio of a negative test & 48.4 & \\
\hline Likelihood ratio of a positive test & $\mathbf{0 . 0 1}$ \\
\hline
\end{tabular}

\section{Future Work}

This assay can be extended to any target that can be recognized by an aptamer. To demonstrate this, we performed preliminary work on an additional target, gluten. Briefly, aptamers that target gluten were chosen by SELEX and screened to hybridize to anchor sequences as described for the P1-16 aptamer. The selected aptamer (GN5) exhibited high sensitivity to gluten, as shown with a dose-dependent curve 
(Figure 7A) showing the fluorescence intensity significantly decreasing in the presence of $0.2 \mathrm{ppm}$ gluten. We also challenged the GN5 aptamer against commercially-available foods and were able to detect the presence of gluten in commonly consumed foods (Figure 7B).

\section{Discussion}

Here we describe the design and development of an aptamer-based assay platform with the potential to detect allergens and other targets in a broad spectrum of foods. For detection of peanut antigen, an aptamer, P1-16, was identified and binding affinity determined for pure peanut protein Ara $\mathrm{h} 1\left(\mathrm{~K}_{\mathrm{d}} \sim 54 \pm\right.$ $5.5 \mathrm{nM})$ and Ara $\mathrm{h} 1$ in peanut butter and peanut flour $\left(\mathrm{K}_{\mathrm{d}} \sim 141 \pm 21.9 \mathrm{ppm}\right.$, and $144 \pm 31.4 \mathrm{ppm}$, respectively; Figure 1). We effectively immobilized P1-16 via anchors to optically clear glass (Figure 2) within a single-use reaction capsule that is run on a consumer-friendly instrument (SI Figure 3a). Specificity of the assay was determined (Figures 3,5 ) and the reagents tested for stability (SI Figure 5). Finally, the assembled peanut allergen test detected peanut antigen in 50 foods spanning 14 food categories (Table 1).

In a recent Joint Expert Consultation meeting by FAO/WHO on Risk Assessment of Food Allergens, an Expert Committee established threshold levels in foods of priority allergens below which most allergic consumers would not suffer an adverse reaction. Based on data reported by Remington et al. $(2020)^{26}$ and Houben et al. (2020) ${ }^{27}$, the Committee recommended a threshold of $2 \mathrm{mg}$ for peanut protein. Our peanut allergen test builds off traditional SELEX approaches ${ }^{1,15}$ to improve assay sensitivity, selectivity, and flexibility ${ }^{28}$ and allow detection of $12.5 \mathrm{ppm}$ peanut protein in foods (equivalent to $37.5 \mu \mathrm{g}$ within a $100 \mathrm{mg}$ food sample tested in the pod), despite the challenges of antigen detection in complex matrices, e.g., high protein, high salt, or acidity. Key to the approach is use of two different anchor sequences, (1) the test anchor, which competes with peanut protein for binding to P1-16 and (2) a control anchor that binds a different region of the P1-16 aptamer and is not competitive with peanut protein binding. The control anchor thus serves as a matrix condition control (Figure 4A). Updating the assay to image the fluorescent intensities of the test and control spots separately allowed the signal output to be normalized ( 1 - intensity of test/intensity of control) to yield a single value that could be compared across a variety of food matrices.

Compared to traditional antibody-based approaches, our aptamer assay features several practical advantages, including reagents that are (1) synthesized and chemically modified in a fast, reproducible, and scalable process; (2) small and inexpensive to manufacture with reproducible production characteristics; and (3) stable over a range of temperatures and $\mathrm{pH}$ values. The all-in-one detection platform also represents an end-to-end solution, involving (1) sample collection, (2) homogenization and filtration methods, including a universal extraction step that can be applied to food as well as other matrices (serum, saliva, etc.), and (3) a precision optical sensor and algorithm with built-in controls. As an added advantage, we demonstrate that our aptamer approach can be readily modified to detect 
additional targets, such as gluten, and is suited to rapid transition to additional food allergens as well as other targets such as pesticides and even heavy metals.

There are 32 million 29,30 people with food allergies in the United States, and allergic reactions lead to approximately 200,000 emergency department visits ${ }^{31}$ and 200 deaths each year ${ }^{32}$. Strict avoidance of allergens in the diet is the sole treatment for food allergies, but due to the ubiquity of the most common allergens in the food chain the risk of accidental exposure is high ${ }^{33-35}$. Food allergy management requires individuals and caregivers to continuously manage exposure to allergens ${ }^{36}$, and food prepared and consumed outside the home can be in question ${ }^{36}$ due to lack of awareness and knowledge about food allergies among restaurant workers ${ }^{37}$. Due to these dangers, children and families experience psychological, social, and economic burdens and caregivers of children with food allergies often experience diminished quality of life, anxiety, and frustration over lack of food allergy awareness ${ }^{38}$. The proposed assay platform can empower consumers to easily and quickly assess the presence of allergens in foods before eating to help people with food allergies and intolerances manage their daily lifestyle of practicing avoidance.

\section{Materials \& Methods}

Selection/description of aptamers/SELEX

A random 76-mer library (random region of 30 nucleotides flanked by 23 nucleotide primer regions) was subjected to 10 rounds of positive SELEX with decreasing concentrations of gluten (Sigma-Aldrich), followed by 7 rounds of counter SELEX against mixtures of proteins including common wheat replacements. The pool was isolated and amplified at the end of each round. At the end of 17 rounds, the enriched pool was sent for sequencing. Twelve of the top hits were synthesized and evaluated, with GN5 being selected for best sensitivity and specificity.

Affinity Measurements

Fluorescence polarization (FP) was used to determine dissociation constants $\left(K_{d} s\right)$ for PT-31, P1-10, P116 , P2-8, and P2-18 interaction with potential targets. The aptamers were synthesized from Integrated DNA Technologies with a Texas Red fluorophore attached to its 5 ' end in order to measure changes in fluorescence polarization. Each experiment was performed on a TECAN Spark $10 \mathrm{M}$ plate reader (excitation $570 \mathrm{~nm} / \mathrm{emission} 625 \mathrm{~nm}$ ) set to 5 kinetic cycles. Samples were prepared in $50 \mathrm{uL}$ with FP buffer (50 mM Tris-HCl, 0.1\% Tween-20, pH 9) containing $5 \mathrm{nM}$ aptamer and increasing concentration of purified AraH1 (Indoor Biotechnologies) or peanut matrix (Teddie brand unsalted peanut butter or Protein Plus brand roasted natural peanut flour) ranging from 0 to $50 \mathrm{uM}$ and incubated for 10 minutes prior to reading on the spectrofluorometer. Peanut matrices were prepared by homogenizing samples at a stock concentration of 100,000 parts per million in FP buffer and clarifying by centrifugation at 5,000 x g for three minutes. Nonlinear regression analyses were used to determine $K_{d} s$ (Prism 8, GraphPad). 
CY5-labeled aptamers and 40 short DNA anchors with a ten-oligonucleotide sequence complementary to the aptamers were synthesized at Integrated DNA Technologies. Half of the anchor sequences contained a poly-A tail and all anchor sequences contained an amine linker at the $5^{\prime}$ end of the oligonucleotide. Each anchor was spotted on epoxy silane-treated slides at various concentrations (1-40 uM) at Applied Microarrays (Tempe, Arizona). Each slide was pre-blocked with $1 \%$ bovine serum albumin in HEPES for two minutes prior to incubation of CY5-aptamer/peanut flour mixture. Aptamer was mixed with peanut flour at different concentrations and allowed to incubate in binding buffer prior to loading onto the well. After a two-minute incubation with mild shaking on an orbital rotator, the slides were washed with binding buffer and scanned for fluorescent CY5 signal. After selection of the 5'-(Amine-6C)-anchor, the linker was extended by six additional carbon atoms and printed at $5 \mathrm{uM}$ concentration on epoxy silane-treated slides for confirmation.

Assay details

COP chips were placed in an air-tight chamber with fluidic channels connected to reservoirs from which wash solution or filtered food homogenate were drawn via a pump system. First, $100 \mathrm{uL}$ of wash solution (20 mM Trizma base, 0.2\% Brij-L4, 0.2\% Capstone FS-31, 0.25 $\mathrm{mM} \mathrm{MgCl}_{2}$ ) was delivered to the chamber, followed by a short air purge. Then, $100 \mathrm{uL}$ of test sample (containing homogenization buffer $(20 \mathrm{mM}$ EPPS, 0.2\% Brij-58, 2\% PEG 8000, 2\% Pluronic F-127, pH 8.4), $15 \mathrm{nM} \mathrm{AF647-P1-16,} \mathrm{and} 30 \mathrm{mM} \mathrm{MgCl}_{2}$ ) was delivered to the chamber at a rate of $1000 \mathrm{uL} / \mathrm{min}$. The chamber was cleared, an image captured, and the intensity of the control spots assessed. If less than $30 \mathrm{rfu}$, another aliquot of $100 \mathrm{uL}$ of test sample was delivered. The process was repeated until the intensity of the control spots is greater than 30 rfu. Then the chamber was washed with $200 \mathrm{uL}$ of wash solution and imaged.

Guard band study

For the matrix interference studies, $15 \mathrm{nM}$ AF647-P1-16 was incubated briefly with the listed additives and components in homogenization buffer (20 mM EPPS, 2\% Pluronic F-127, 2\% PEG 8000, 0.2\% Brij-58, pH 8.4). The percentage represents the amount in a food sample, meaning for a value of $100 \%, 0.1 \mathrm{~g}$ of component was added to $3 \mathrm{~mL}$ of assay buffer. Peanut flour was then added to the $50 \mathrm{ppm}$ samples, and the assay was run as described above.

Specificity studies

AF647-P1-16 aptamer (20 nM) was incubated with increasing concentrations of purified AraH proteins (Indoor Biotechnologies, AraH1, \#NA-AH1-1; AraH2, \#NA-AH2-1; AraH3, \#NA-AH3-1; AraH6, \#NA-AH6-1; AraH8, \#RP-AH8-1) in assay buffer and $30 \mathrm{mM} \mathrm{MgCl}_{2}$. Commercially available nut flours (pecan, walnut, pistachio, hazelnut, almond, sunflower, and cashew) were homogenized with assay buffer and clarified by centrifugation at $5,000 \times \mathrm{g}$ for three minutes. To study the specificity of P1-16 aptamer when tree nut is 
present, 0 or 50 ppm peanut flour was spiked into clarified 50 ppm tree nut flour in assay buffer or $0.1 \%$ non-fat milk (dry powder, American Bio). Samples were assayed as described for the guard band study.

Matrix testing to validate assay

Chips were printed with both $12.5 \mathrm{uM} \mathrm{P1-16}$ anchor and $7 \mathrm{uM}$ of the control anchor. Foods were sampled at $0.1 \mathrm{~g}$ and homogenized for $45 \mathrm{~s}$ in $3 \mathrm{~mL}$ of assay buffer with $7.5 \mathrm{nM} \mathrm{P1-16.} \mathrm{For} \mathrm{peanut} \mathrm{containing}$ samples, $30 \mathrm{uL}$ of a $5000 \mathrm{ppm}$ peanut homogenate was also added. Finally, $23 \mathrm{mM} \mathrm{MgCl} 2$ was added to all samples. The assay ran as described above. The foods tested were: vanilla ice cream, sugar-free vanilla wafer, gelato, milk chocolate, mint chocolate chip ice cream, nacho cheese, pasta sauce, mushroom soup, sweetened cereal, white chocolate, applesauce, oat cereal, chicken gravy, hoisin sauce, packaged cupcakes, rice noodles, vanilla crispy squares, blue cheese dressing, alfredo sauce, frosting, pink meringue cookie, fluff, marshmallow cereal, sauerkraut, fruit flavored chewy candy, Asian dressing, fruit punch, coconut milk, coffee creamer, flavored tortilla chips, French dressing, corn chips, sweetened cereal, electrolyte beverage, granola, honey, chocolate-covered wafer, mashed potatoes, olive oil, pear baby food, rainbow sherbet, ranch dressing, white rice, shortbread cookie, sweet red chili sauce, tomato soup, chocolate-covered cookie bar, and yogurt.

\section{Gluten assay}

GN5 aptamer was incubated in gluten assay buffer (GAB, $15.4 \mathrm{mM}$ MES buffer, $0.08 \%$ Tween-20, 30\% ethanol, and $1 \mathrm{mM} \mathrm{MgCl}_{2}, \mathrm{pH}$ 5) with increasing concentrations of gluten. Gluten (wheat source, Sigma Life Science) was extracted in GAB and diluted in GAB with $20 \mathrm{nM}$ GB1. For the food testing, commercially available foods were paired with the closest match for the gluten-free counterpart: wheat round crackers versus gluten-free round crackers (corn starch and rice flour); wheat frosted blueberry toaster pastry versus gluten-free frosted blueberry toaster pastry (rice flour); wheat pretzel sticks versus gluten free pretzel stick (corn and potato starches); country white bread versus gluten-free white bread (pea, tapioca, and rice starches); animal crackers (wheat) versus gluten-free animal crackers (pea and potato starches) were tested with GN5. Each food was prepared as described in the matrix testing description; however after filtration, the food filtrate was diluted by an additional 1:10 with GAB. For both assays, $250 \mathrm{uL}$ of GAB is delivered to the chamber, followed by a short air purge. Then, $500 \mathrm{uL}$ of test sample (GAB and $20 \mathrm{nM} \mathrm{GN5}$, with or without gluten) is delivered to the chamber at a rate of 1000 $\mathrm{uL} / \mathrm{min}$. Then the chamber was washed with $525 \mathrm{uL}$ of $\mathrm{GAB}$ with $10 \mathrm{mM} \mathrm{MgCl}_{2}$ with the same flow rate. The chips were then air dried and imaged.

Long-term stability experiments

AF647-P1-16 (10 nM) was formulated in autoclaved homogenization buffer (20 mM EPPS, 0.2\% Brij-58, $2 \%$ PEG-8000, 2\% Pluronic F-127, pH 8.4) under aseptic, sterile environmental conditions within a clean room facility. Aliquots of such samples were subjected to accelerated aging at $37^{\circ} \mathrm{C}$. At each time point, samples were compared with respect to age-matched fresh P1-16. 


\section{Declarations}

\section{Author Contributions}

S.S., V.V., and A.G. conceived and planned the experiments, analyzed the results, and wrote the manuscript. S.S., V.V., V.C., O.A., and L.Y. carried out the experiments. A.G. supervised the project. W.S., J.S., D.F., and H.S. aided in the interpretation of the results. All authors discussed and results and commented on the manuscript.

\section{Competing Interests Statement}

S.S., V.V., V.C., O.A., L.Y., and A.G. were employed by DOTS Technology Corp during the work. A.G. is a cofounder and member of the board of directors. W.S., J.S., D.F., and H.S. are members of the scientific advisory board.

\section{References}

1. Ellington, A. D. \& Szostak, J. W. In vitro selection of RNA molecules that bind specific ligands. Nature 346, 818-822 (1990).

2. Zhao, L. et al. Aptamers and Aptasensors for Highly Specific Recognition and Sensitive Detection of Marine Biotoxins: Recent Advances and Perspectives. Toxins 10, 427 (2018).

3. Zhang, Y., Lai, B. \& Juhas, M. Recent Advances in Aptamer Discovery and Applications. Molecules 24, 941 (2019).

4. Zhuo, Z. et al. Recent Advances in SELEX Technology and Aptamer Applications in Biomedicine. International Journal of Molecular Sciences 18, 2142 (2017).

5. Thiviyanathan, V. \& Gorenstein, D. G. Aptamers and the next generation of diagnostic reagents. PROTEOMICS - Clinical Applications 6, 563-573 (2012).

6. Ni, X., Castanares, M., Mukherjee, A. \& Lupold, S. E. Nucleic Acid Aptamers: Clinical Applications and Promising New Horizons. Current Medicinal Chemistry 18, 4206-4214 (2011).

7. Dunn, M. R., Jimenez, R. M. \& Chaput, J. C. Analysis of aptamer discovery and technology. Nature Reviews Chemistry 1, 0076 (2017).

8. Voskuil, J. Commercial antibodies and their validation. F1000Research 3, 232 (2014).

9. Bamrungsap, S. et al. Pattern Recognition of Cancer Cells Using Aptamer-Conjugated Magnetic Nanoparticles. ACS Nano 6, 3974-3981 (2012). 
10. Catuogno, S. \& Esposito, C. L. Aptamer Cell-Based Selection: Overview and Advances. Biomedicines 5, 49 (2017).

11. Al-Ahmed, N., Alsowaidi, S. \& Vadas, P. Peanut Allergy: An Overview. Allergy, Asthma \& Clinical Immunology 4, 139 (2008).

12. Sicherer, S. H., Warren, C. M., Dant, C., Gupta, R. S. \& Nadeau, K. C. Food Allergy from Infancy Through Adulthood. Journal of Allergy and Clinical Immunology: In Practice 8, 1854-1864 (2020).

13. Sicherer, S. H., Muñoz-Furlong, A. \& Sampson, H. A. Prevalence of peanut and tree nut allergy in the United States determined by means of a random digit dial telephone survey. Journal of Allergy and Clinical Immunology 112, 1203-1207 (2003).

14. Davis, J. \& Dean, L. Peanut Composition, Flavor and Nutrition. in 289-345 (2016). doi:10.1016/B978-1-63067-038-2.00011-3.

15. Tuerk, C. \& Gold, L. Systematic evolution of ligands by exponential enrichment: RNA ligands to bacteriophage T4 DNA polymerase. Science 249, 505-510 (1990).

16. Palladino, C. \& Breiteneder, H. Peanut allergens. Molecular Immunology 100, 58-70 (2018).

17. Zhou, Y. et al. Peanut Allergy, Allergen Composition, and Methods of Reducing Allergenicity: A Review. International Journal of Food Science 2013, 1-8 (2013).

18. Gijsbers, A., Nishigaki, T. \& Sánchez-Puig, N. Fluorescence Anisotropy as a Tool to Study Proteinprotein Interactions. Journal of Visualized Experiments (2016) doi:10.3791/54640.

19. Ciulli, A. Biophysical Screening for the Discovery of Small-Molecule Ligands. in 357-388 (2013). doi:10.1007/978-1-62703-398-5_13.

20. Cabanos, C. et al. Crystal structure of the major peanut allergen Ara h 1. Molecular Immunology 49, 115-123 (2011).

21. Chruszcz, M. et al. Structural and Immunologic Characterization of Ara h 1, a Major Peanut Allergen. Journal of Biological Chemistry 286, 39318-39327 (2011).

22. Jin, T., Guo, F., Chen, Y., Howard, A. \& Zhang, Y.-Z. Crystal structure of Ara h 3, a major allergen in peanut. Molecular Immunology 46, 1796-1804 (2009).

23. Mueller, G. A., Maleki, S. J. \& Pedersen, L. C. The Molecular Basis of Peanut Allergy. Current Allergy and Asthma Reports 14, 429 (2014).

24. Berlier, J. E. et al. Quantitative Comparison of Long-wavelength Alexa Fluor Dyes to Cy Dyes: Fluorescence of the Dyes and Their Bioconjugates. Journal of Histochemistry \& Cytochemistry 51, 16991712 (2003). 
25. Geiselhart, S., Hoffmann-Sommergruber, K. \& Bublin, M. Tree nut allergens. Molecular Immunology 100, 71-81 (2018).

26. Remington, B. C. et al. Updated population minimal eliciting dose distributions for use in risk assessment of 14 priority food allergens. Food and Chemical Toxicology 139, (2020).

27. Houben, G. F. et al. Full range of population Eliciting Dose values for 14 priority allergenic foods and recommendations for use in risk characterization. Food and Chemical Toxicology 146, (2020).

28. Song, S., Wang, L., Li, J., Fan, C. \& Zhao, J. Aptamer-based biosensors. TrAC Trends in Analytical Chemistry 27, 108-117 (2008).

29. Gupta, R. S. et al. Prevalence and Severity of Food Allergies Among US Adults. JAMA network open 2, (2019).

30. Gupta, R. S. et al. The public health impact of parent-reported childhood food allergies in the United States. Pediatrics 142, (2018).

31. Clark, S., Espinola, J., Rudders, S. A., Banerji, A. \& Camargo, C. A. Frequency of US emergency department visits for food-related acute allergic reactions. Journal of Allergy and Clinical Immunology 127, 682-683 (2011).

32. Fda \& CarrotNewYork. Food Facts From the U.S. Food and Drug Administration. http://www.fda.gov/food/ingredientspackaginglabeling/foodallergens/ucm079311.htm (2017).

33. Gern, J. E., Yang, E., Evrard, H. M. \& Sampson, H. A. Allergic Reactions to Milk-Contaminated "Nondairy" Products. New England Journal of Medicine 324, 976-979 (1991).

34. Jones, R. T., Squillace, D. L. \& Yunginger, J. W. Anaphylaxis in a milk-allergic child after ingestion of milk-contaminated kosher-pareve-labeled "dairy-free" dessert. Annals of allergy 68, 223-227 (1992).

35. LAOPRASERT, N. et al. Anaphylaxis in a Milk-Allergic Child Following Ingestion of Lemon Sorbet Containing Trace Quantities of Milk. Journal of Food Protection 61, 1522-1524 (1998).

36. Muñoz-Furlong, A. Daily Coping Strategies for Patients and Their Families. Pediatrics 111, 1654 (2003).

37. Kwon, J. \& Lee, Y. M. Exploration of past experiences, attitudes and preventive behaviors of consumers with food allergies about dining out: A focus group study. Food Protection Trends 32, 736746 (2012).

38. Springston, E. E. et al. Variations in quality of life among caregivers of food allergic children. Annals of Allergy, Asthma \& Immunology 105, 287-294.e3 (2010). 

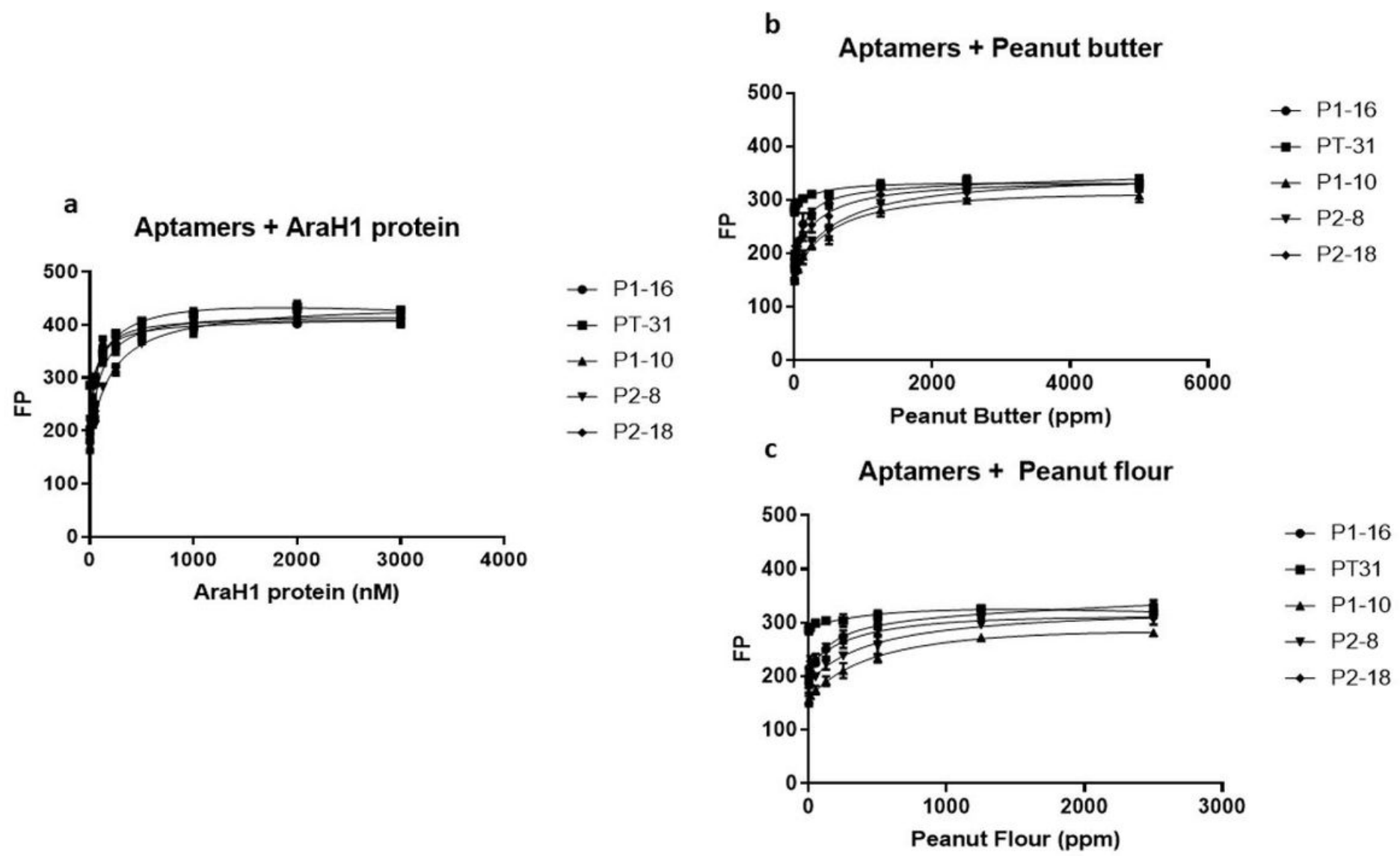

Figure 1

Determination of dissociation constants (Kds) for five peanut aptamers and targets. Five aptamers were incubated with increasing concentrations of target purified Ara $\mathrm{h} 1$ protein to determine the Kd by fluorescence polarization. (A) Purified Ara h 1, (B) Peanut butter, (C) Peanut flour. Five independent replicates were tested, and fitting of the binding isotherm yielded Kd values shown in SI Table 1. Error bars represent the standard deviation of the mean. 

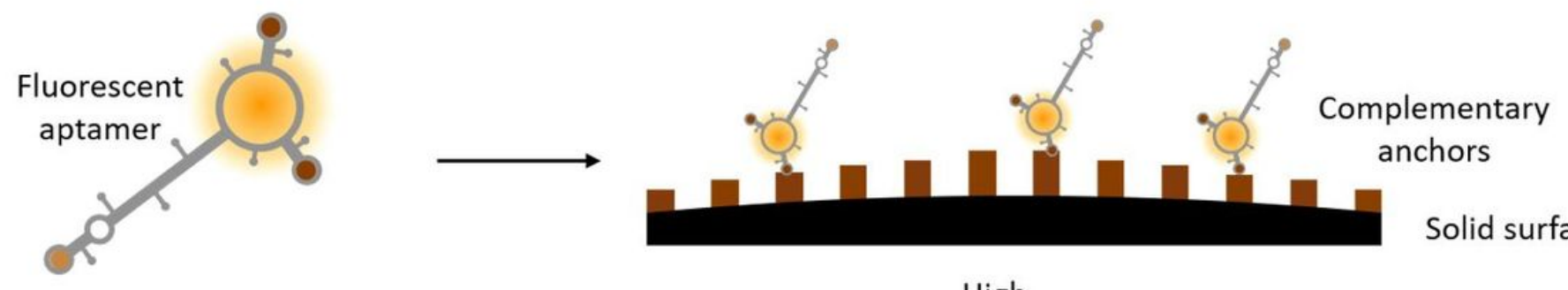

Solid surface

High

fluorescence
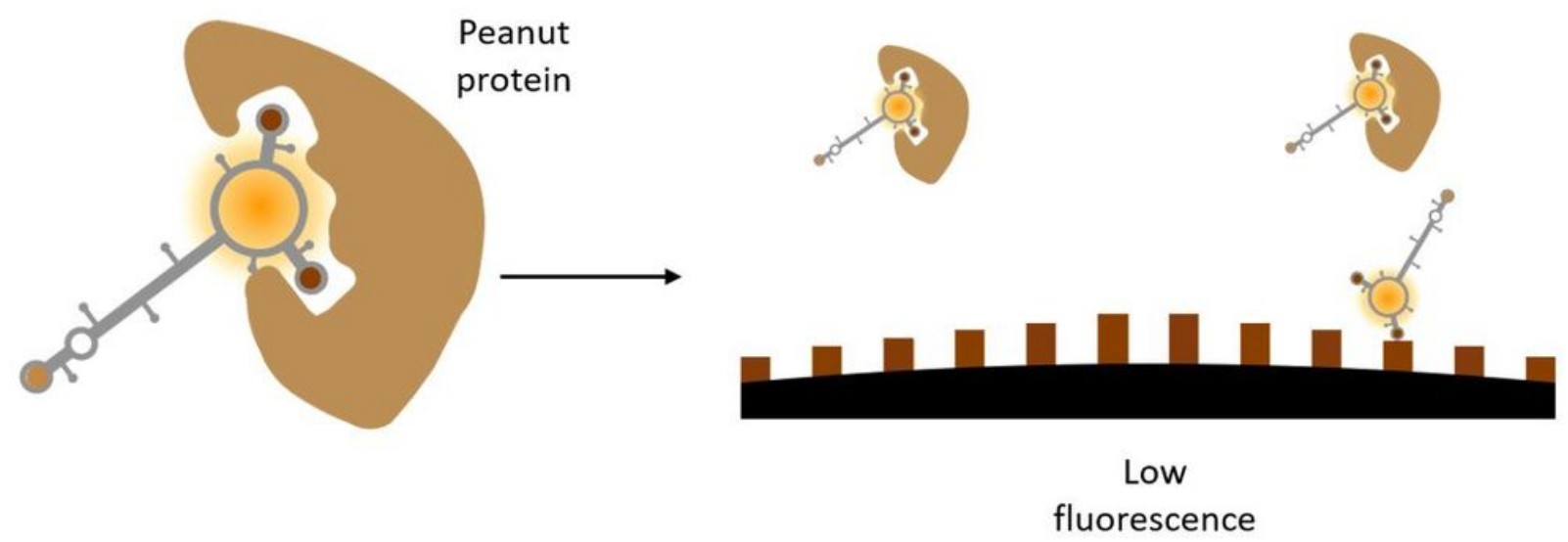

Figure 2

Principles of the assay. Fluorescently labeled aptamer is incubated with sample. If it binds its target, it cannot bind its complementary anchor on the solid surface, leading to low fluorescence.

\section{P1-16 aptamer + AraH proteins}

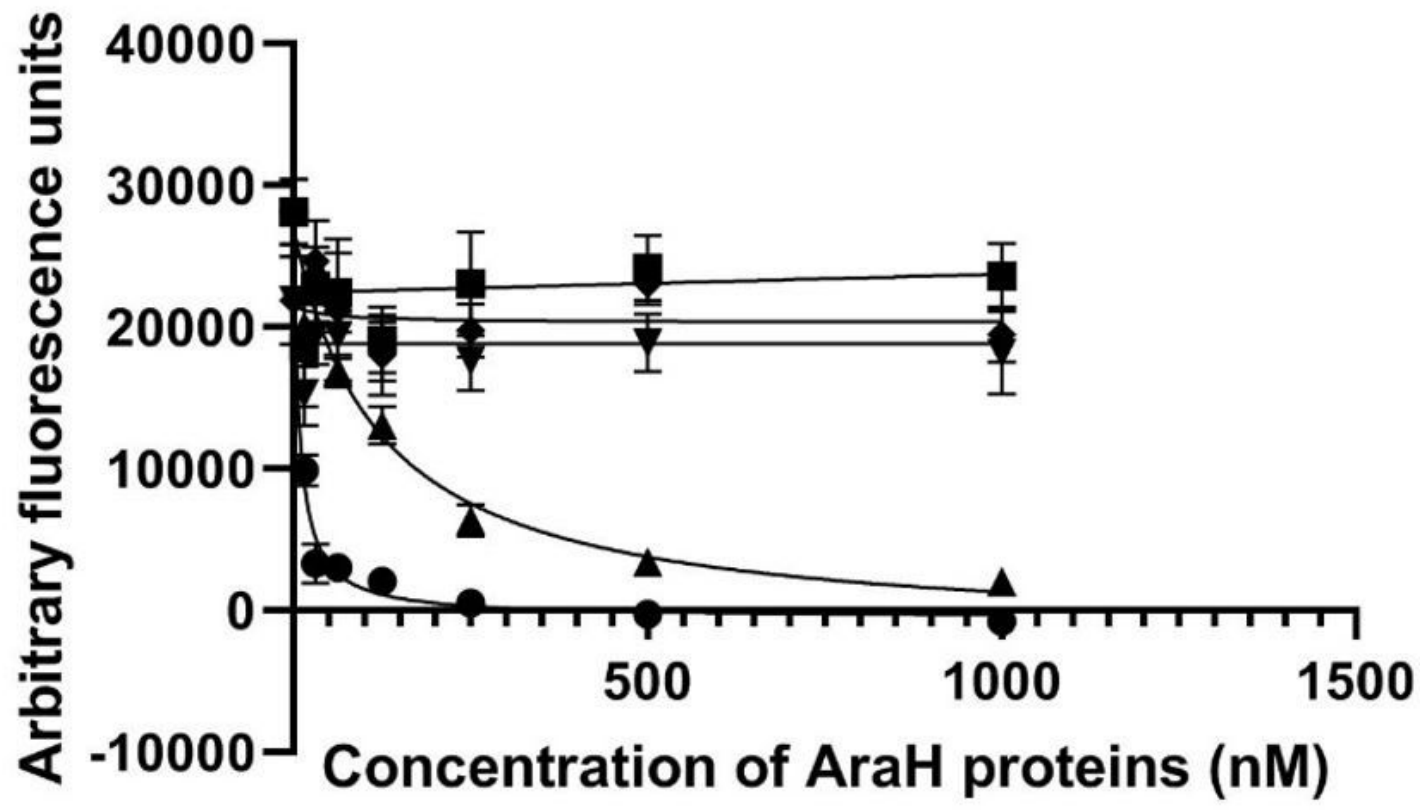

$\rightarrow$ AraH1

$-\mathrm{AraH} 2$

- AraH3

$\rightarrow$ AraH6

$\bullet$ AraH8 


\section{Figure 3}

Alexa Fluor 647-labeled P1-16 aptamer binds to Ara $\mathrm{h} 1$ and Ara $\mathrm{h} 3$. Binding specificity was assessed by incubating purified AraH proteins, Ara h 1, Ara h 2, Ara h 3, Ara h 6, and Ara h 8, with AF647-P1-16 aptamer and testing with the benchtop assay. Curve fitting was performed using non-linear regression analysis. Four replicates were tested for each concentration with error bars representing the standard deviation of the mean.

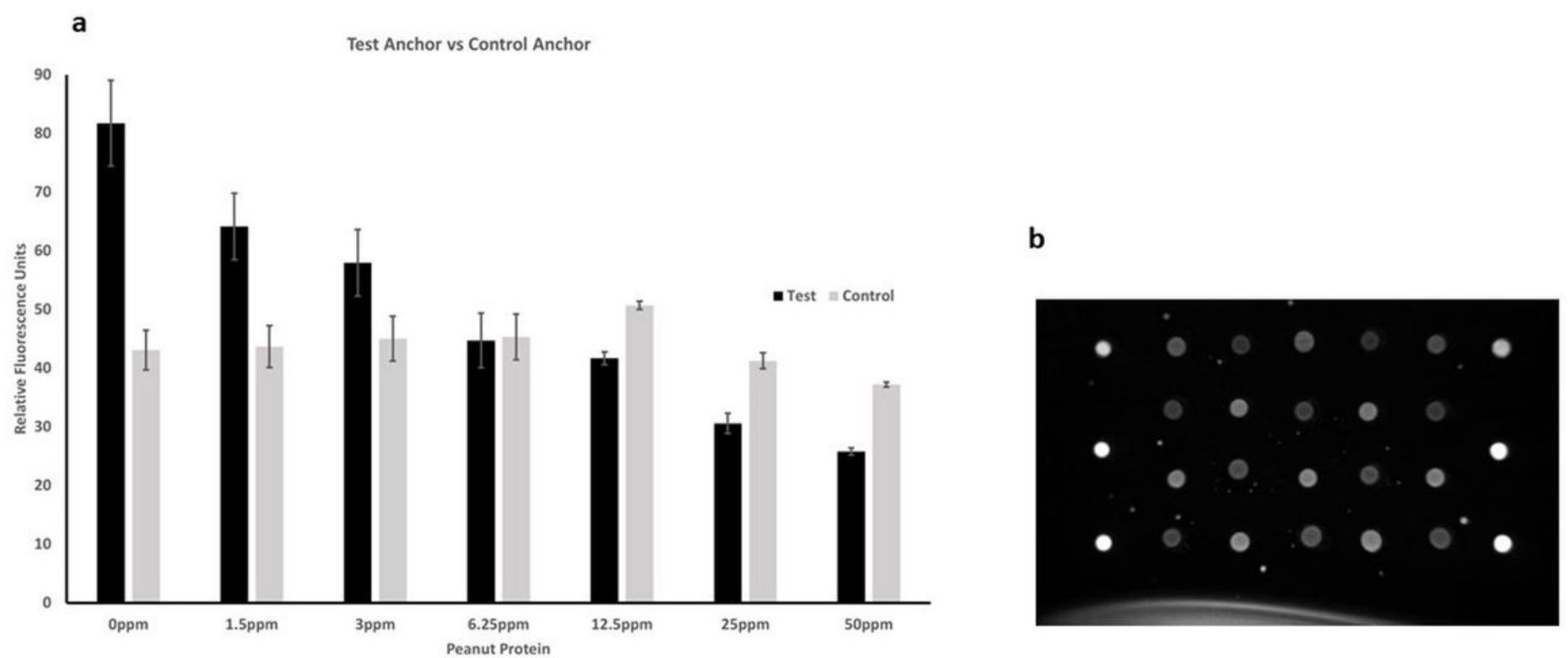

Figure 4

AF647-P1-16 aptamer binds to control anchor with or without peanut in the sample. (A) The comparison of P1-16 aptamer binding to two different anchors (test and control) spotted on the same surface was assessed by incubating P1-16 aptamer with increasing concentrations of clarified peanut flour homogenate. Five replicates of each peanut protein concentration were tested. Error bars represent the standard error of the mean. (B) Representative image of Alexa Fluor 647-P1-16 aptamer bound to both the test spots (top left and alternating) and the control spots. The brighter spots on the left and right sides represent alignment markers for optical performance. 

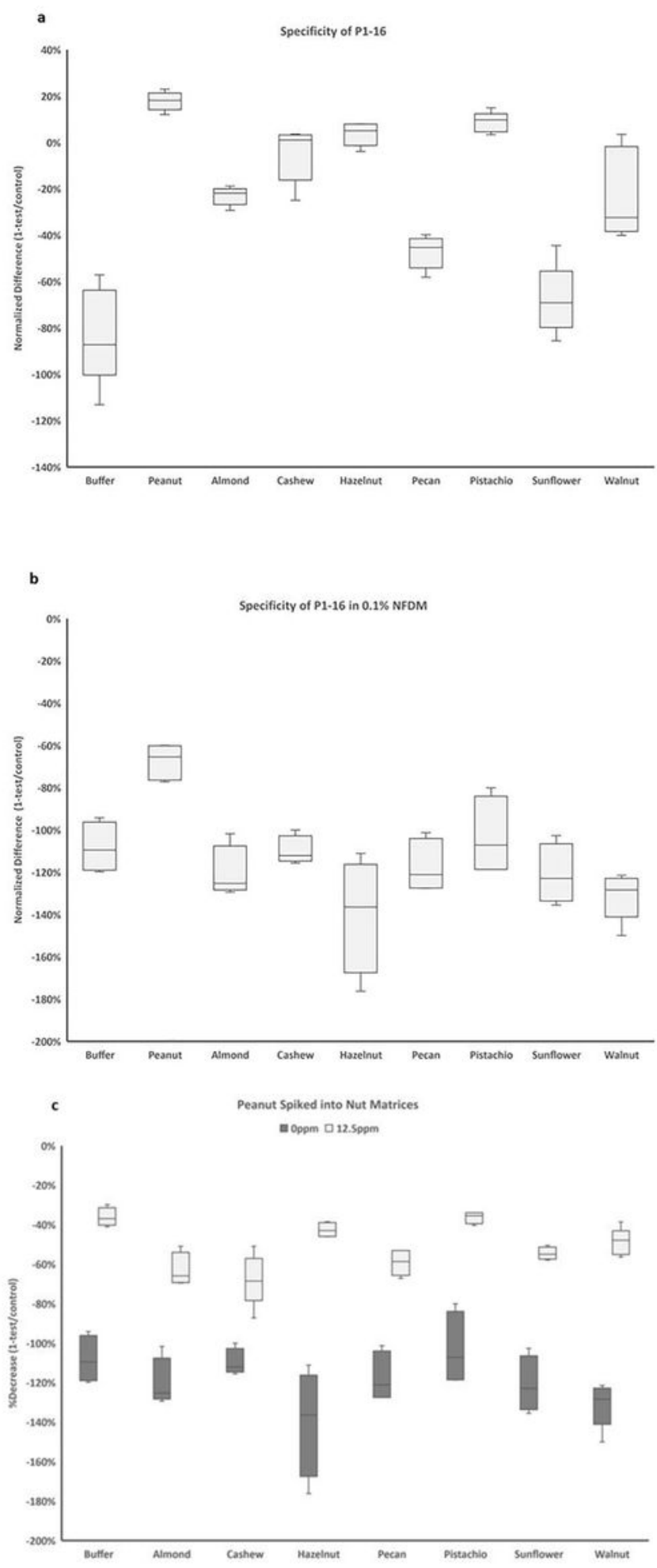

\section{Figure 5}

Specificity against tree nuts. (A) P1-16 aptamer binds to peanut protein(s) preferentially to tree nuts. P116 is sensitive to tree nuts in a concentration-dependent manner. P1-16 aptamer was incubated in clarified peanut or tree nut flours blended in assay buffer. (B) $0.1 \%$ milk added to the buffer. (C) P1-16 aptamer was incubated with clarified tree nut homogenate at $50 \mathrm{ppm}$ nut flour (or control buffer) and spiked with 0 or $12.5 \mathrm{ppm}$ peanut protein. Four or five replicates were tested for each concentration. 

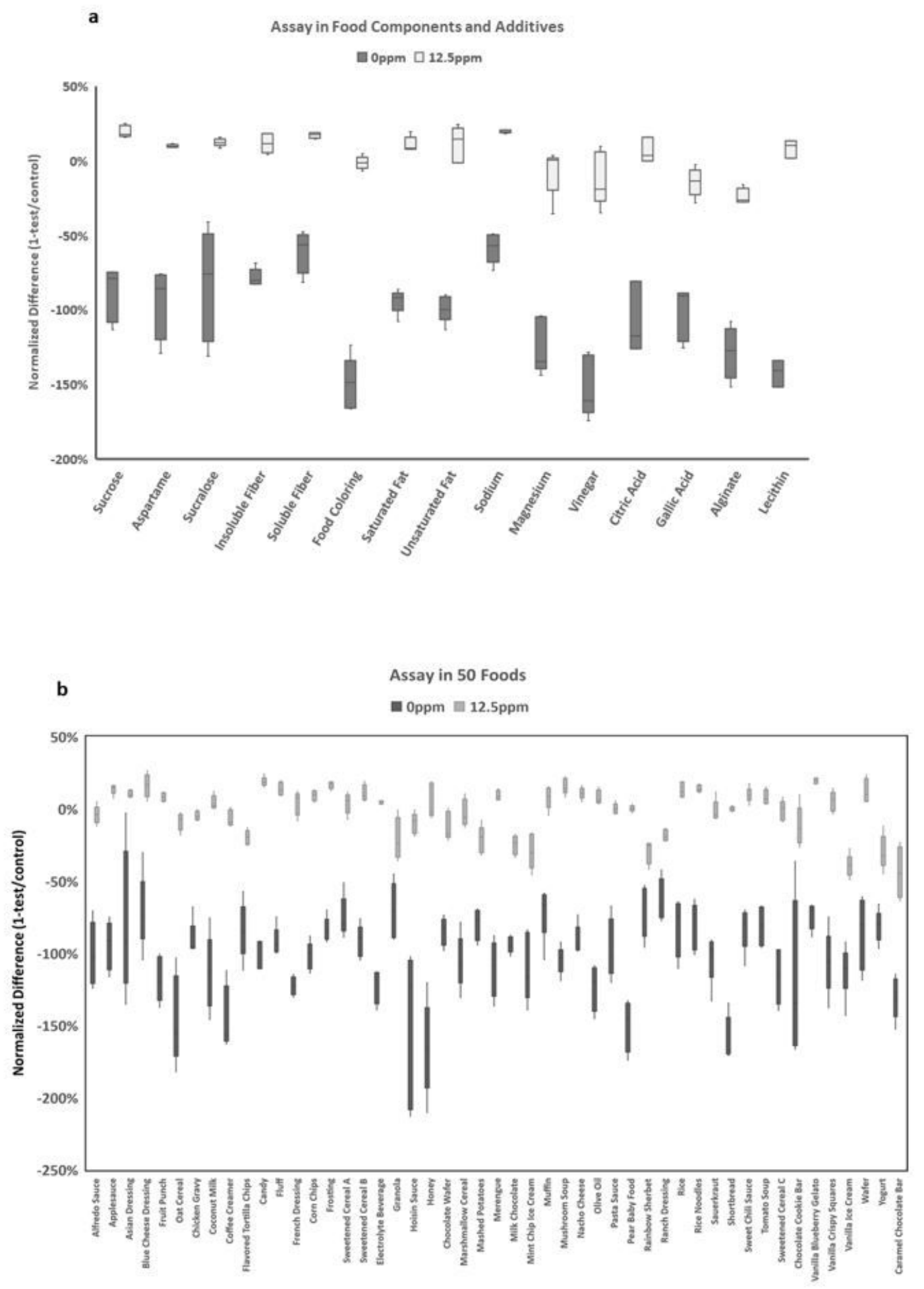

Figure 6

Assay Validation. (A) Peanut can be detected in major food components and common food additives. Assay was run using multiple food components and additives, both with and without $12.5 \mathrm{ppm}$ of peanut protein. Four or five replicates of each peanut flour concentration were tested. (B) Food samples with and without peanut protein can be differentiated by comparing intensity of test spots to control spots. Fifty commercially available foods, spiked with $0 \mathrm{ppm}$ or $12.5 \mathrm{ppm}$ peanut protein, at least 5 replicates each, 
were tested with AF647-P1-16 aptamer. All samples are plotted by normalized difference (1 test/control).

a

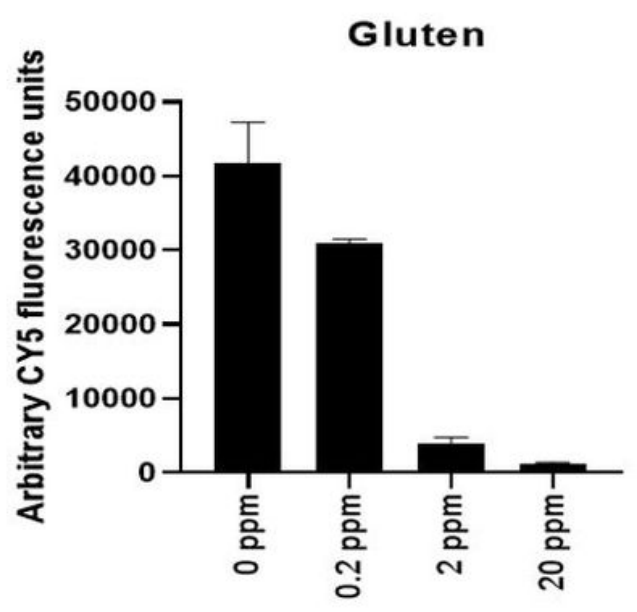

b

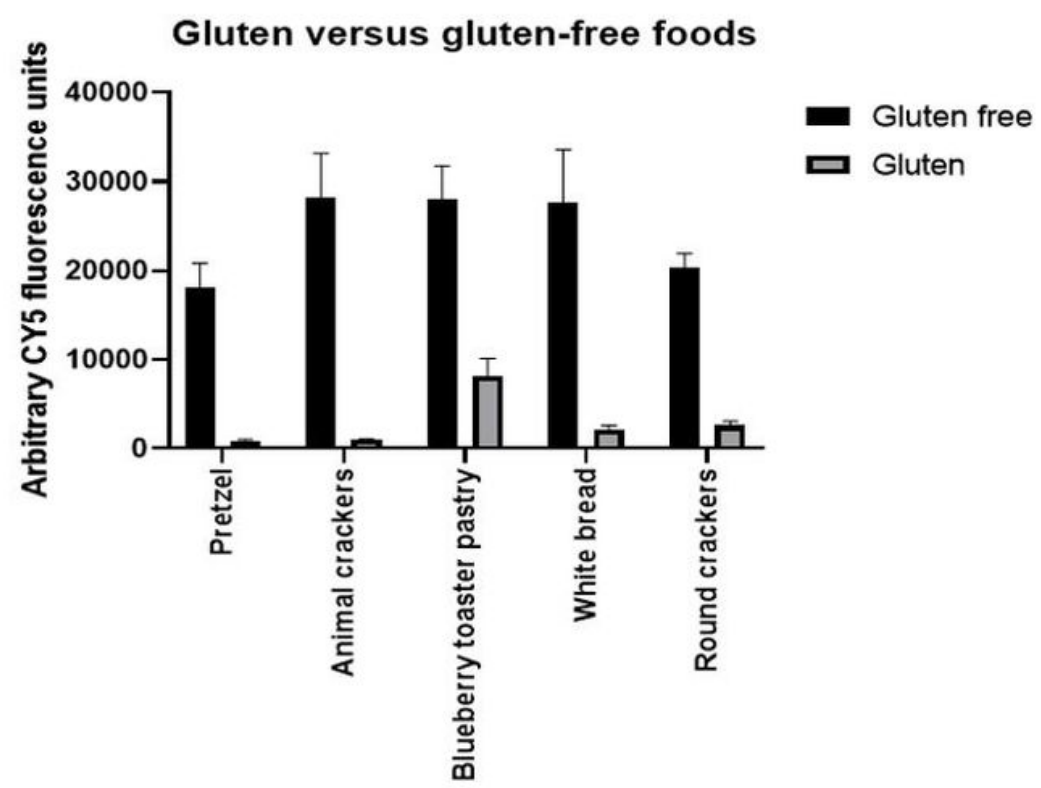

Figure 7

Future Work. (A) CY5-GN5 aptamer binds to gluten in a concentration dependent manner and in a variety of food matrices. GN5 aptamer was incubated in buffer spiked with increasing concentrations of gluten. (B) Commercially available foods (gluten versus gluten-free) were homogenized, filtered, then incubated with GN5. As described for the P1-16 aptamer, the samples were incubated with a chiplet spotted with a 10 oligonucleotide anchor complementary to sequence of GN5. Four replicates of each sample were tested. Error bars represent the standard deviation of the mean.

\section{Supplementary Files}

This is a list of supplementary files associated with this preprint. Click to download.

- ManuscriptSI111521.pdf 